\title{
Correspondence
}

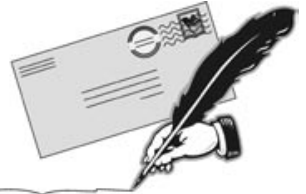

\section{Anesthesiologists and the \\ pharmaceutical industry: awareness is paramount}

To the Editor:

We believe the Proceedings of the Canadian Consensus Conference $^{1}$ on the emerging (as mentioned in the text) role of recombinant factor VIIa (rFVIIa) in onpump cardiac surgery to control perioperative bleeding raise three important points for discussion.

First, in disparity with the randomized controlled trials (RCTs) (only two) published on the use of activated recombinant human factor VII (rHuFVIIa) in cardiac surgery, the Conference did not restrict its conclusions to non-coronary on-pump cardiac surgery but seemingly included all on-pump cardiac surgeries. The level of risk of arterial thrombosis in patients receiving $\mathrm{rHuFVIIa}$ after coronary artery bypass grafting $(\mathrm{CABG})$ remains unknown. The Conference issues a strong recommendation against the use of rHuFVIIa as a prophylaxis for excessive blood loss (grade 1B), and a weak one (grade 2C) in favour of its use as a rescue therapy. The therapy "might be effective" but is associated with a "troublingly high rate of thromboembolic complications and mortality". Not mentioned is that rHuFVIIa displayed prothrombotic effects in three animal studies published recently (albeit two were not available by December 2006). The first, from Mazer's group, unfortunately only available in abstract form ${ }^{2}$ showed that the infusion of rHuFVIIa $\left(300 \mu \mathrm{g} \cdot \mathrm{kg}^{-1}\right)$ leads to venous and arterial graft thrombosis in rabbit neck vessel preparations. Since the Conference met in June 2006, Samama's ${ }^{3}$ and our group ${ }^{4}$ have shown that even at lower doses (150 and $100 \mu \mathrm{g} \cdot \mathrm{kg}^{-1}$ respectively), the infusion of rHuFVIIa increases the incidence of thromboembolic events and of total occlusion ${ }^{4}$ in rabbit carotid arteries subjected to an endothelial lesion and a reduction of flow. Thus, the experimental evidence now clearly points towards a thrombogenic role for rHuFVIIa. The conditions of these experiments resemble those of cardiac surgery (exposure of the subendothelium and variable flow conditions) and suggest an increased risk of graft occlusion. A rigorously conducted RCT will be essential before issuing convincing recommendations on the use of rHuFVIIa in cardiac surgery, specially for the specific CABG indication.
Second, despite the commendable disclosure statement by the authors, we fear that some of the content of this publication may reflect the fact that the Conference was largely sponsored by the manufacturer of the drug. The purpose of a disclosure statement is to warn the reader against thinking that he is about to receive unbiased, fair and balanced information. Yet, no matter its size, any retribution will influence the physician in favour of the sponsor's interests. ${ }^{5}$ The participants may have been inclined to insist, in their introductory comments, on the burden of excessive bleeding in patients undergoing cardiac surgery, thereby promoting the potential benefits of rHuFVIIa. While the potential for the drug to induce thrombosis is presented, its implications are not fully discussed. Despite the systematic exclusion of all patients at risk, a significant increase of arterial thrombotic events has been reported when investigating the treatment of intracerebral hemorrhage with rHuFVIIa. ${ }^{6}$ The Conference recommends that patients at risk of thromboembolic complications be excluded: but are these not those patients with vascular (particularly coronary) disease?

Third, the collaboration between physicians and the industry is vital for drug development in Canada: physicians provide essential resources whereas, in return of government extending patent protection, research drug pharmaceutical companies commit to allocate $10 \%$ of sales to Canadian-based research. This situation raises a number of challenges that are in the process of being resolved. ${ }^{7}$ As we become fully conscious of the influence of marketing imperatives of pharmaceutical companies, a number of practices will change. In the meantime, heightened awareness will assist anesthesiologists (and we include ourselves in this statement) in filtering the information that is presented to them.

François Hélie PhD

Jean-François Hardy MD FRCPC

CHUM Hôpital Notre-Dame, Montreal, Canada

E-mail: jean-francois.hardy@umontreal.ca

Disclosure statement: François Hélie held manager positions with Genizon BioSciences, Montreal (Genetics, Clinical Research; 2002 to 2005) and Bayer HealthCare Pharmaceuticals, Toronto (Medical \& Scientific Affairs; 2005 to 2007). 
Jean-François Hardy has, since 1984, received funding from the following pharmaceutical companies: Janssen Pharmaceuticals (research funding), Sterling Drugs (research funding), Kabi Pharmacia (research funding), Ibex Technologies (research funding), Hemosol Inc. (lecturing and research funding), Ortho Biotech (lecturing and research funding), Bayer Inc (lecturing and research funding), Novo Nordisk (lecturing). JeanFrançois Hardy is Chairman of the Data Monitoring Committee of the ongoing study on rHuFVIIa in cardiac surgery sponsored by Novo Nordisk and, as such, cannot engage in any marketing activities (e.g., lectures, symposia, etc.) of that company.

Accepted for publication September 20, 2007.

\section{References}

I Karkouti K, Beattie WS, Crowther MA, et al. The role of recombinant factor VIIa in on-pump cardiac surgery: proceedings of the Canadian Consensus Conference. Can J Anesth 2007; 54: 573-82.

2 ChChina TS, Lapierre H, Jackson ZS, et al. Effect of recombinant factor VIIa on rabbit vascular graft patency. Can J Anesth 2004; 51: Al (abstract).

3 Godier A, Mazoyer E, Cymbalista F, Cupa M, Samama $C M$. Recombinant activated factor VII efficacy and safety in a model of bleeding and thrombosis in hypothermic rabbits: a blind study. J Thromb Haemost 2007; 5: 244-9.

4 Charbonnean S, Girard F, Boudreault D, Ruel M, Blais $N$, Hardy JF. Recombinant human activated factor VII is thrombogenic in a rabbit model of cyclic flow reduction and does not reduce intra-abdominal bleeding. Thromb Haemost 2007; 97: 296-303.

5 Blumenthal D. Doctors and drug companies. N Engl J Med 2004; 351: 1885-90.

6 Mayer SA, Brun NC, Begtrup K, et al. Recombinant activated factor VII for acute intracerebral hemorrhage. N Engl J Med 2005; 352: 777-85.

7 Lewis S, Baird P, Evans RG, et al. Dancing with the porcupine: rules for governing the university-industry relationship. CMAJ 2001; 165: 783-5.

\section{Reply:}

We appreciate the interest in, and criticism of, our consensus conference proceedings on the role of activate recombinant buman factor VII (rHuFVIIa) by Drs. Hélie and Hardy. We fully agree with their principal argument that, in academic medicine, financial conflicts of interest are pervasive and problematic, and there needs to be heightened awareness of this issue. ${ }^{2}$ Whether our report was unduly influenced (i.e., biased) by the panel members' financial conflicts of interest (which were clearly outlined in the report), as is suggested by Drs. Hélie and Hardy, however, is debatable.

Drs. Hélie and Hardy suggest that we may have exaggerated the burden of excessive blood loss to promote the benefits of rHuFVIIa. In our report, we simply stated that excessive blood loss "remains an important complication of on-pump cardiac surgery." We supported this statement by reporting its current incidence at Canadian institutions, ${ }^{3}$ and cited four studies that suggest that "excessive blood loss, rather than simply being a marker for surgical misadventure as it is often presumed, is independently associated with both morbidity and mortality." These comments are not only scientifically sound, but, in our opinion, are also "fair and balanced."

They also suggest that, in formulating our recommendations, we failed to properly consider the prothrombotic risks of rHuFVIIa. It is intriguing that they support this argument by noting that we did not consider three animal studies that show rHuFVIIa is prothrombotic in a model of vessel injury in non-coagulopathic rabbits. This is intriguing because: 1) these experiments were not published in full by the cut-off date for inclusion in our review; 2) they do not mention a similar experiment which found that $r H u F V I I$ a decreased bleeding without increasing arterial thrombosis in rabbits; ${ }^{4}$ and 3) there is an implicit suggestion that the findings of animal experiments should supersede clinical evidence.

Drs. Hélie and Hardy are also concerned that we did not restrict our conclusions to non-coronary on-pump cardiac surgery, and correctly point out that "the level of risk of arterial thrombosis in patients receiving $r H u F-$ VIIa after coronary artery bypass grafting (CABG) remains unknown." In formulating our recommendations, we paid particular attention to the potential risks of rHuFVIIa, and were fully cognizant of the limitations of the existing safety data in cardiac surgery. Our concerns are noted throughout the manuscript, and are clearly outlined in our concluding remarks: "Recombinant factor VIIa is a potent hemostatic drug, and as such, has clear rishs of thromboembolic complications. Much work remains in delineating its risk-benefit profile in the cardiac surgery setting." Specifically, we did not restrict our conclusions to non-coronary onpump cardiac surgery because the panel concluded that potential benefits of $r H u F V I I a$ likely outweigh its risks in patients who develop massive, refractory hemorrhage, irrespective of whether they underwent CABG. To illustrate this point, data from our cardiovascular surgery database (which we have previously used to assess the risk of blood product transfusions $)^{5-7}$ reveal that while 
the mortality rate in $C A B G$ patients who receive $\geq 15$ $U$ of red blood cells is $42 \%$, the rate is only $16 \%$ in those who receive 5- $10 \mathrm{U}$ and have a myocardial infarction. Thus, the risk-benefit profile of rHuFVIIa is likely to be favourable even under assumptions of modest efficacy and maximal risk.

To conclude, in the spirit of heightening the awareness of clinicians to the issue of conflicts of interest in biomedical research, we would like to offer the following excerpt from an excellent article by Dr. Bekelman et al..2 "Conflicts of interest are a set of conditions in which professional judgment concerning a primary interest (such as a patient's welfare or validity of research) tends to be unduly influenced by a secondary interest (such as financial gain). Financial interests are not the only, or necessarily the most powerful, secondary interests faced by investigators and academic institutions. For investigators, other pressures, including the desire for professional recognition and the need to compete successfully for research funding, are intrinsic to the research process.”

Keyvan Karkouti MD FRCPC MSC

Toronto General Hospital, University of Toronto, Toronto, Canada

E-mail: keyvan.karkouti@uhn.on.ca

On behalf of the panel members of the Canadian Consensus Conference on the role of recombinant factor VIIa in on-pump cardiac surgery.

\section{References}

1 Karkouti K, Beattie WS, Crowther MA, et al. The role of recombinant factor VIIa in on-pump cardiac surgery: proceedings of the Canadian Consensus Conference. Can J Anesth 2007; 54: 573-82.

2 Bekelman JE, Li $\Upsilon$, Gross CP. Scope and impact of financial conflicts of interest in biomedical research: a systematic review. JAMA 2003; 289: 454-65.

3 Karkouti K, Wijeysundera D, Beattie WS, Yau T. Variability and predictability of blood product use in cardiac surgery: a multicentre study. Transfusion 2007; doi:10.1111/j.1537-2995.2007.01432.x.

4 Fattorutto M, Tourreau-Pham S, Mazoyer E, et al. Recombinant activated factor VII decreases bleeding without increasing arterial thrombosis in rabbits. Can J Anesth 2004; 51: 672-9.

5 Karkouti K, Yau TM, Riazi S, et al. Determinants of complications with recombinant factor VIIa for refractory blood loss in cardiac surgery. Can J Anesth 2006; 53: 802-9.

6 Karkouti K, Wijeysundera DN, Yau TM, et al. Platelet transfusions are not associated with increased morbidity or mortality in cardiac surgery. Can J Anesth 2006; 53:
279-87.

7 Karkouti K, Wijeysundera DN, Yau TM, et al. The independent association of massive blood loss with mortality in cardiac surgery. Transfusion 2004; 44 : 1453-62.

\section{Interference of bispectral index monitoring with intraoperative use of the electromyograph endotracheal tube}

To the Editor:

The electromyograph endotracheal tube (EMG ETT) allows monitoring of the recurrent laryngeal nerve (RLN) to help reduce nerve injury ${ }^{1}$ during thyroid surgery. The Xomed EMG ETT (Medtronic ENT, Jacksonville, FL, USA) (Figure) consists of an oral endotracheal tube integrated with paired wire electrodes that are exposed for $30 \mathrm{~mm}$ at the glottis level allowing contact with the vocal cords. ${ }^{2}$ Spontaneous EMG activity is monitored and the surgeon can use a stimulating probe to test whether an anatomic structure is in fact the RLN. Bispectral index monitoring (BIS) has been introduced to clinical anesthesia as a monitor of the "wakefulness" of the patient. While the device generally gives reliable information, electrical devices have been known to interfere with the accuracy of the BIS value. ${ }^{3}$ We present a case of the EMG ETT erroneously increasing the BIS values.

A 48-yr-old woman (ASA physical status I, 60 $\mathrm{kg}$ ) underwent total thyroidectomy for treatment of a thyroid nodule. A trigger-free general anesthetic technique was chosen because of a possible family history of malignant hyperthermia. Anesthesia was induced with propofol, midazolam, and sufentanil. The trachea was easily intubated without aid of muscle relaxants using the Xomed EMG ETT. Anesthesia was maintained with nitrous oxide, oxygen, and intravenous infusions of propofol and sufentanil. The initial BIS value after induction was 28 and remained at approximately 40 for the next $30 \mathrm{~min}$ of surgical preparation. No muscle relaxants were used during the surgery so that the integrity of the RLN could be tested using the EMG ETT. The start of surgical exploration was accompanied by a sudden increase in the BIS value to 80 . During this time the heart rate, blood pressure, and end-tidal $\mathrm{CO}_{2}$ remained normal and constant. The propofol and sufentanil infusions were quickly increased and midazolam $4 \mathrm{mg}$ in bolus was also given. The BIS value remained at $70-80$ for the next $30 \mathrm{~min}$ in spite of the above measures and an additional $18 \mathrm{mg}$ of etomidate $i v$. The EMG stimulator was turned off at the completion of surgery and 